\title{
The physiological basis of neuromuscular fatigue during high intensity exercise
}

\section{AUTHORS:}

Faryal Zahir*1, Radha Budhwar², Gabrielle Gonsalves ${ }^{3}$, Lily Green ${ }^{4}$ and Aliza Barua ${ }^{3}$.

[where * indicates the primary author.]

\section{INSTITUTION:}

1. Department of Biology, McMaster University, Hamilton, ON

2. Department of Health Sciences, McMaster University, Hamilton, ON

3. Department of Science, McMaster University, Hamilton, ON

4. Department of Arts \& Science, McMaster University, Hamilton, ON

EDITOR'S NOTE: The following was recognized as a winning project in the McMaster Indicium research competition and recommended for publication (as a graphical abstract) within the STEM Fellowship Journal. The Indicium research competition was the first undergraduate research conference hosted by one of STEM Fellowship's university branches.

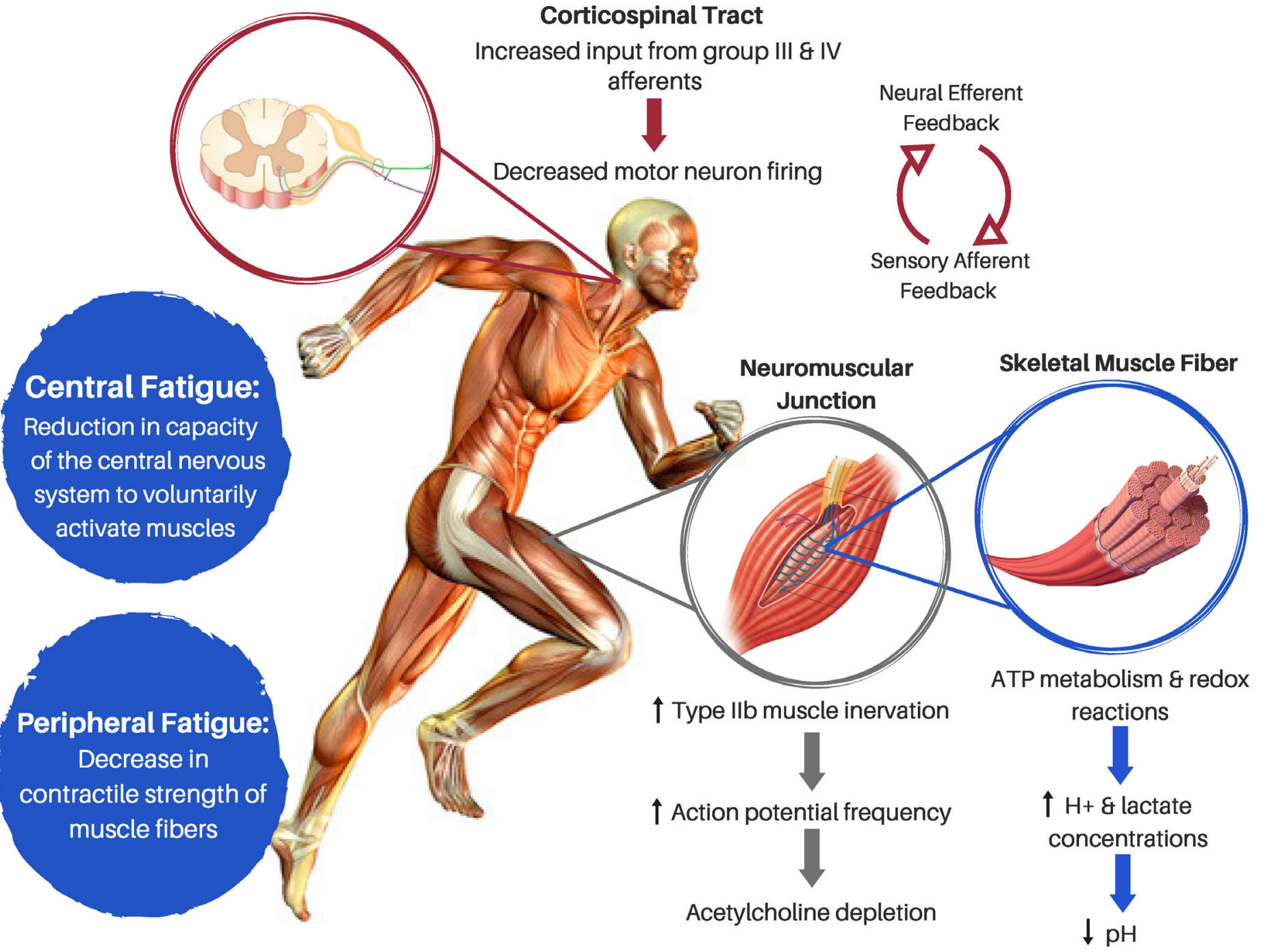




\section{Abstract}

Introduction:

Neuromuscular fatigue refers to a reduction in maximal force generation capacity, and is categorized as central and peripheral. Central fatigue is defined as a reduction in the ability of the central nervous system to voluntarily activate muscles, and peripheral fatigue indicates a decrease in the contractile strength of muscle fibers. During high intensity exercise, motor neurons are involved in the recruitment of type IIB muscle fibers as they are fast-twitch, high glycolytic, and have low aerobic capacity. Furthermore, group III and IV muscle afferents detect the physiological circumstances in the body and convey signals to the brain that influence the onset of central and peripheral fatigue.

\section{Methods:}

A PRISMA flow diagram was created to record relevant studies found from scholarly databases. Inclusion criteria required studies from 2005 to 2017, and subject grouping headings required key terms indicating that the presence of central and peripheral fatigue was analyzed on healthy adult subjects performing exercise. To ensure that high quality studies were analyzed, each article was independently rated using the National Institute of Health Quality Assessment Tool criteria.

\section{Discussion:}

During low intensity exercise, asynchronous motor unit recruitment is involved in delaying the onset of muscle fatigue. However, this is not apparent in high intensity exercises, as maximal motor unit firing is required in order to sustain a maximal level of force output. Persistent firing of action potentials to maintain muscle contraction results in acetylcholine depletion at the motor end plate, initiating the process of central fatigue. Furthermore, due to prolonged metabolite accumulation in skeletal muscle fibers, group III and IV afferents convey signals to the motor cortex and cause a reduction in the action potential conduction velocities along the contracting muscle. This leads to the onset of peripheral fatigue. As high intensity exercise proceeds, electromyogram (EMG) measurements display this as an increase in amplitude to reflect heightened motor unit recruitment and a compressed power density spectrum alongside a decreased centre frequency. This is determined by the innervated muscle fiber's conduction velocity and subsequent variations in the action potential waveform shape.

\section{Conclusion:}

A record of current studies systematically display the overview of muscle fatigue and its underlying mechanisms during exercise. However, further research is yet to be conducted for a more comprehensive understanding regarding the onset and recovery of neuromuscular fatigue in varied population demographics and physiological circumstances. Likewise, the distinctive roles of group III and IV muscle afferents in supraspinal stimulation require further investigation in order to gain a holistic understanding of their involvement in central fatigue and resistance training. Additional research in this subject matter is currently being explored through technology involving imaging studies, as they have potential to elucidate motor cortex activity alongside other regions of the brain and portray neuromuscular muscle fatigue eminently.

\section{Keywords}

neuromuscular fatigue, central fatigue, peripheral fatigue, maximal voluntary contraction, EMG, motor unit, group III/IV muscle afferents, lactate, acetylcholine, high intensity exercise, skeletal muscle 


\section{References}

1. Amann M, Blain G, Proctor L, Sebranek J, Pegelow D, Dempsey J. Implications of group III and IV muscle afferents for high-intensity endurance exercise performance in humans. The Journal of Physiology. 2011;589(21):5299-5309.

2. Amann M. Central and Peripheral Fatigue. Medicine \& Science in Sports \& Exercise. 2011;43(11):2039-2045.

3. Behringer M, Grützner S, Montag J, McCourt M, Ring M, Mester J. Effects of stimulation frequency, amplitude, and impulse width on muscle fatigue. Muscle \& Nerve. 2015;53(4):608-616.

4. Billaut F, Basset F, Falgairette G. Muscle coordination changes during intermittent cycling sprints. Neuroscience Letters. 2005;380(3):265-269.

5. Billaut F, Basset F, Giacomoni M, Lemaître F, Tricot V, Falgairette G. Effect of High-Intensity Intermittent Cycling Sprints on Neuromuscular Activity. International Journal of Sports Medicine. 2006;27(1):25-30.

6. Billaut $F$, Basset $F$. Effect of different recovery patterns on repeated-sprint ability and neuromuscular responses. Journal of Sports Sciences. 2007;25(8):905-913.

7. Bishop D, Edge J. Determinants of repeated-sprint ability in females matched for single-sprint performance. European Journal of Applied Physiology. 2006;97(4):373-379.

8. Boyas S, Guével A. Neuromuscular fatigue in healthy muscle: Underlying factors and adaptation mechanisms. Annals of Physical and Rehabilitation Medicine. 2011;54(2):88-108.

9. Boyas S, Guével A. Neuromuscular fatigue in healthy muscle: Underlying factors and adaptation mechanisms. Annals of Physical and Rehabilitation Medicine. 2011;54(2):88-108.

10. Buchheit M. Fatigue during Repeated Sprints. Sports Medicine. 2012;42(2):165-167.

11. Burnley M, Jones A. Power-duration relationship: Physiology, fatigue, and the limits of human performance. European Journal of Sport Science. 2016;:1-12.

12. Camic C, Housh T, Johnson G, Hendrix C, Zuniga J, Mielke M et al. An EMG frequency-based test for estimating the neuromuscular fatigue threshold during cycle ergometry. European Journal of Applied Physiology. 2009;108(2):337-345.

13. Camic C, Housh T, Johnson G, Hendrix C, Zuniga J, Mielke M et al. An EMG frequency-based test for estimating the neuromuscular fatigue threshold during cycle ergometry. European Journal of Applied Physiology. 2009;108(2):337-345.

14. Decorte N, Lafaix P, Millet G, Wuyam B, Verges S. Central and peripheral fatigue kinetics during exhaustive constant-load cycling. Scandinavian Journal of Medicine \& Science in Sports. 2010;22(3):381-391.

15. Froyd C, Beltrami F, Millet G, Noakes T. Central Regulation and Neuromuscular Fatigue during Exercise of Different Durations. Medicine \& Science in Sports \& Exercise. 2016;48(6):1024-1032.

16. Froyd C, Millet G, Noakes T. The development of peripheral fatigue and shortterm recovery during self-paced high-intensity exercise. The Journal of Physiology. 2013;591(5):1339-1346.

17. Froyd C, Millet G, Noakes T. The development of peripheral fatigue and shortterm recovery during self-paced high-intensity exercise. The Journal of Physiology. 2013;591(5):1339-1346.

18. Gandevia S. Voluntary muscle strength and endurance: 'The mechanism of voluntary muscle fatigue'. Experimental Physiology. 2008;93(9):1030-1033.

19. Girard O, Bishop D, Racinais S. Neuromuscular Adjustments of the Quadriceps Muscle after Repeated Cycling Sprints. PLoS ONE. 2013;8(5):e61793.

20. Glace B, Kremenic I, Ben-Avi S, McHugh M. Central Fatigue in Male and Female Cyclists Evaluated by Peripheral Magnetic Stimulation. Medicine \& Science in Sports \& Exercise. 2008;40(Supplement):S350.

21. Gould J, Cleland B, Mani D, Amiridis I, Enoka R. Motor unit activity in biceps brachii of left-handed humans during sustained contractions with two load types. Journal of Neurophysiology. 2016;116(3):1358-1365.

22. Hureau T, Olivier N, Millet G, Meste 0 , Blain G. Exercise performance is regulated during repeated sprints to limit the development of peripheral fatigue beyond a critical threshold. Experimental Physiology. 2014;99(7):951-963.

23. Hureau T, Olivier N, Millet G, Meste 0 , Blain G. Exercise performance is regulated during repeated sprints to limit the development of peripheral fatigue beyond a critical threshold. Experimental Physiology. 2014;99(7):951-963.

24. Hyungdon Kim. Differential effects of Taekwondo training on postural stability after fatigue induced by aerobic endurance exercise. Exercise Science. 2011;20(3):215-226.

25. Kernozek T, Torry M, Iwasaki M. Gender Differences in Lower Extremity Landing Mechanics Caused by Neuromuscular Fatigue. The American Journal of Sports Medicine. 2008;36(3):554-565.

26. Mastaglia F. The relationship between muscle pain and fatigue. Neuromuscular Disorders. 2012;22:S178-S180.

27. Matkowski B, Place N, Martin A, Lepers R. Neuromuscular fatigue differs following unilateral vs bilateral sustained submaximal contractions. Scandinavian Journal of Medicine \& Science in Sports. 2011;21(2):268-276.

28. McGrath M, Padua D, Lewek M. Evidence Of Central And Peripheral Fatigue After A Functional Fatigue Protocol. Medicine \& Science in Sports \& Exercise. 2009; $41: 292$.

29. McGrath M, Padua D, Lewek M. Evidence Of Central And Peripheral Fatigue After A Functional Fatigue Protocol. Medicine \& Science in Sports \& Exercise. 2009;41:292.

30. Mendez-Villanueva A, Hamer P, Bishop D. Fatigue in repeated-sprint exercise is related to muscle power factors and reduced neuromuscular activity. European Journal of Applied Physiology. 2008;103(4):411-419.

31. Monks M, Compton C, Yetman J, Power K, Button D. Repeated sprint ability but not neuromuscular fatigue is dependent on short versus long duration recovery time between sprints in healthy males. Journal of Science and Medicine in Sport. 2017;20(6):600-605.

32. O'Leary T, Morris M, Collett J, Howells K. Central and peripheral fatigue following non-exhaustive and exhaustive exercise of disparate metabolic demands. Scandinavian Journal of Medicine \& Science in Sports. 2015;26(11):1287-1300.

33. Papaiordanidou M, Hayashibe M, Varray A, Fattal C, Guiraud D. A new method for muscle fatigue assessment: Online model identification techniques. Muscle \& Nerve. 2014;50(4):556-563.

34. Pearcey G, Murphy J, Behm D, Hay D, Power K, Button D. Neuromuscular fatigue of the knee extensors during repeated maximal intensity intermittent-sprints on a cycle ergometer. Muscle \& Nerve. 2015;51(4):569-579.

35. Place N, Maffiuletti N, Martin A, Lepers R. Assessment of the reliability of central and peripheral fatigue after sustained maximal voluntary contraction of the quadriceps muscle. Muscle \& Nerve. 2007;35(4):486-495

36. Ranieri F, Di Lazzaro V. The role of motor neuron drive in muscle fatigue. Neuromuscular Disorders. 2012;22:S157-S161.

37. Sadri K, Khani M, Sadri I. Role of Central Fatigue in Resistance and Endurance Exercises: An Emphasis on Mechanisms and Potential Sites. Sportlogia. 2014;10(2):65-80.

38. Taylor J, Amann M, Duchateau J, Meeusen R, Rice C. Neural Contributions to Muscle Fatigue. Medicine \& Science in Sports \& Exercise. 2016;48(11):2294-2306.

39. Yoon T, Schlinder Delap B, Griffith E, Hunter S. Mechanisms of fatigue differ after low- and high-force fatiguing contractions in men and women. Muscle \& Nerve. 2007;36(4):515-524.

40. Ørtenblad N, Westerblad H, Nielsen J. Muscle glycogen stores and fatigue. The Journal of Physiology. 2013;591(18):4405-4413. 